\title{
$H$ pylori infection is associated with downregulation of E-cadherin, a molecule involved in epithelial cell adhesion and proliferation control
}

\author{
A M Terrés, J M Pajares, D O’Toole, S Ahern, D Kelleher
}

\begin{abstract}
Extracellular matrix proteins and proteins involved in epithelial adhesion are essential for maintenance of tissue structure. Helicobacter pylori is the major aetiological agent in peptic ulcer disease and has been shown to increase gastric cancer risk up to ninefold. In this study, changes induced by $H$ pylori on the expression of extracellular matrix proteins (collagen IV, fibronectin, and laminin) as well as two essential proteins for cell-basement and cell-cell adhesion ( $\alpha 6$ integrin and E-cadherin) were assessed. Immunohistochemistry was performed in antral biopsy sections obtained from infected and non-infected patients, and light microscopy was used to determine the distribution and intensity of specific staining. The results showed that the infection was significantly associated with downregulation of E-cadherin, an essential protein for maintenance of solid tissues and differentiation, but did not induce changes in the expression of a6-integrin or the extracellular matrix proteins.

(F Clin Pathol 1998;51:410-412)
\end{abstract}

Keywords: E-cadherin; Helicobacter pylori; gastric cancer

Department of Clinical Medicine, Trinity College Dublin,

Republic of Ireland

A M Terrés

D O'Toole

$S$ Ahern

D Kelleher

Department of

Gastroenterology,

Hospital de la

Princesa, Universidad

Autónoma de Madrid,

Spain

J M Pajares

Correspondence to:

Dr Ana M Terrés,

Department of Clinical

Medicine, SPD Research

Laboratories, St James

Hospital, Dublin 8, Republic

of Ireland; email

amterres@tcd.ie

Accepted for publication

13 January 1998
It is currently accepted that Helicobacter pylori ( $H$ pylori) is the main factor in the complex pathogenesis of the peptic ulcer, ${ }^{1}$ and there is also recent evidence relating the infection to the development of gastric cancer. ${ }^{2}$ The pathological mechanisms involved in these processes begin with $H$ pylori penetration of the mucus layer and adhesion to the underlying gastric epithelium. ${ }^{3}$ Cell adhesion in conjunction with the production of surface urease, lipase, and protein toxins may induce rapid destruction of epithelial cells, exposing subepithelial tissues and the extracellular matrix. Bacteria can attach to the basement membrane by high affinity binding to collagen IV and laminin, ${ }^{4}$ which favours the neutrophilic infiltration of the ulcer crater. $H$ pylori has also been shown to promote epithelial cell hyperproliferation, ${ }^{5}$ a necessary step in the preliminary stages of the development of gastric carcinoma.

In this study we analysed the influence of $H$ pylori infection on the expression of proteins from the extracellular matrix, such as laminin, fibronectin, and collagen IV, as well as proteins involved in epithelial cell adhesion, $\alpha 6$-integrin and E-cadherin. Epithelial basement membranes are important determinants of cell differentiation, cell modelling, and tissue repair. ${ }^{6}$ Laminin and collagen IV help to bind epithelial cells to the basal lamina, while fibronectin helps to bind the matrix macromolecules and connective tissue cells on the opposite side. ${ }^{7}$ Integrins appear to act as the major receptors for cell attachment in the extracellular matrices. Considering the intrinsic function of these proteins, a reduced expression of either of them in the stomach could facilitate the desquamation of the epithelial cell layer, favouring ulceration of the gastric tissue that is associated with $H$ pylori infection.

We also assessed the expression of E-cadherin, a calcium dependent protein involved in cell-cell adhesion at the level of the adherent junctions in the epithelium. ${ }^{8}$ Continued expression and functional activity of E-cadherin are required for cells to remain tightly associated in the epithelium, and in its absence many other proteins involved in this process become incapable of supporting intercellular adhesion. Owing to its capacity to maintain the state of adhesion between epithelial cells, this molecule is important in tissue differentiation and maintenance, and it is thought to act as an important suppressor of epithelial tumour cell invasion and metastatic spread. ${ }^{9}$ As there is a strong association between $H$ pylori and increased risk of gastric carcinoma, we aimed to determine whether $H$ pylori might play a role in altered expression of E-cadherin.

\section{Methods}

TISSUE IMMUNOSTAINING

Thirty patients with gastrointestinal symptoms were included in the study. All patients underwent gastroduodenoscopy, and routine biopsy specimens were taken from the gastric antrum for histological diagnosis and urease test. An additional biopsy was taken in order to perform immunohistochemical assays. $H$ pylori was considered to be present when the rapid urease test and histological diagnosis after staining with giemsa were positive. When both tests were negative, the patient was considered to be $H$ pylori negative. Fourteen patients were not infected by the bacteria ( 13 had normal gastric mucosa and one had chronic gastritis) and the remaining 16 were infected with $H$ pylori (13 

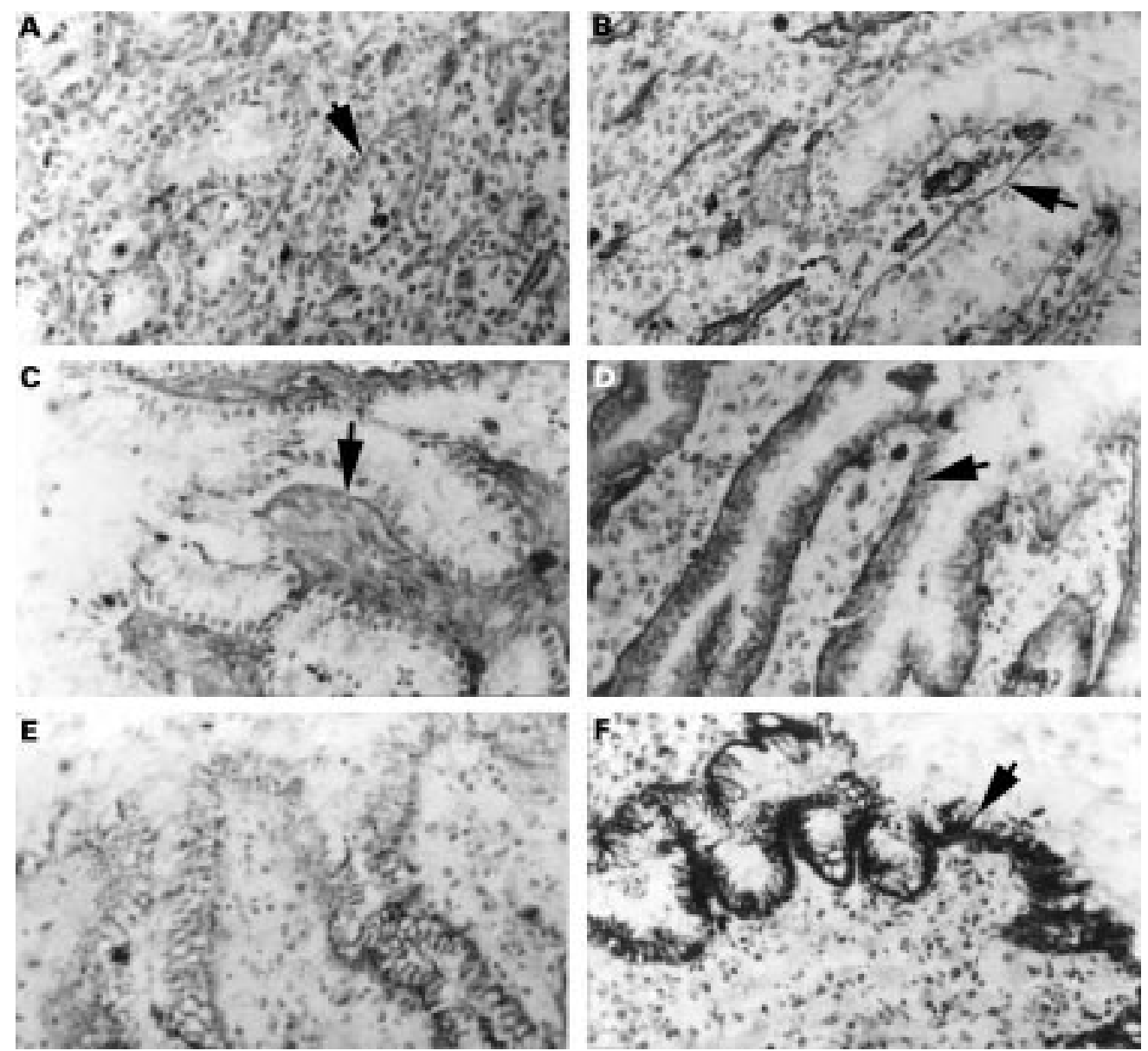

Figure 1 Immunohistochemical staining of gastric mucosa. Analysis was carried out in 30 patients (16 H pylori positive and $14 \mathrm{H}$ pylori negative), and density of staining was assessed blindly and scored by two independent observers without knowledge of the $H$ pylori status. The $\chi^{2}$ test was used for data analysis. H pylori infection was not associated with any significant changes on the expression of laminin $(A)$, collagen $I V(B)$, fibronectin $(C)$, or a6-integrin $(D)$. However, the expression of $E$-cadherin was significantly reduced in infected samples $(E)$ compared with normal tissue $(F)(p<0.001, n$ $=30) . \times 364$.

chronic gastritis, two duodenal ulcer, and one gastric ulcer).

The study was approved by the human ethics committee of St James Hospital, Dublin.

Biopsies for immunohistochemical staining were processed as described elsewhere. ${ }^{10}$ Briefly, biopsies were immediately snap frozen in nitrogen cooled isopentane and stored at $-70^{\circ} \mathrm{C}$. Gastric sections $(5 \mu \mathrm{m})$ were obtained with a $-25^{\circ} \mathrm{C}$ cooled cryostat, air dried, acetone fixed, and stained by the peroxidaseantiperoxidase method. ${ }^{11}$ To visualise the binding of the monoclonal antibodies, sections were developed with Graham-Karnovsky solution containing $0.5 \mathrm{mg} / \mathrm{ml}$ of 3-3'diaminobenzidine tetrahydrochloride (DAB) (Sigma) and hydrogen peroxide. Sections were counterstained with Carazzi's haematoxylin, dehydrated, and mounted by routine methods. Murine hybridoma anti-HLA-IE (American tissue culture collection, ATCC) was used as negative control.

Tissue sections were examined blindly by two independent observers who did not know the $H$ pylori status of the samples and graded them as strong or weak staining. Data were statistically analysed by the $\chi^{2}$ test.
MONOCLONAL ANTIBODIES

The following monoclonal antibodies (mAb) were used: anti-fibronectin (Dako, Golstrup, Denmark; dilution 1:1000), anticollagen IV (Dako; dilution 1:100), anti- $\alpha 6$-integrin (the kind gift of Dr Sánchez-Madrid, Spain; obtained as undiluted supernatant fluids of the hybridoma), anti-laminin (BRL, Ireland; dilution 1:100), and anti-E-cadherin (Transduction Laboratories, Lexington, Kentucky, USA; $1: 50)$.

\section{Results}

Tissue expression of collagen IV, laminin, and fibronectin was first assessed. Laminin and collagen IV coexpressed in the basal lamina immediately beneath the epithelial layer (fig 1A and B), while fibronectin was observed dispersed on the lamina propria in all samples (fig $1 C)$. No variation was observed in the level of expression or distribution in any of these proteins in infected samples compared with normal samples. Semiquantitative data are shown in table 1 .

Expression of molecules involved in epithelial adhesion was then analysed. $\alpha 6$-Integrin was expressed on the basal membrane of the epithelial cells (fig 1D). Very strong staining 
Table 1 Semiquantitative assessment of protein expression

\begin{tabular}{|c|c|c|c|c|c|c|c|}
\hline & \multirow[b]{2}{*}{ Location } & \multicolumn{2}{|c|}{ Strong staining } & \multicolumn{2}{|c|}{ Weak staining } & \multirow[b]{2}{*}{$p$ Value } & \multirow[b]{2}{*}{$n$} \\
\hline & & $H P+$ & $H P-$ & $H P+$ & $H P-$ & & \\
\hline E-cadherin & Epithelium & $25.0 \%$ & $83.2 \%$ & $75.0 \%$ & $16.8 \%$ & $<0.001$ & 30 \\
\hline a6-Integrin & Epithelium & $72.5 \%$ & $80.0 \%$ & $27.5 \%$ & $20.0 \%$ & NS & 30 \\
\hline Collagen IV & Basement membrane & $100 \%$ & $100 \%$ & - & - & NS & 30 \\
\hline Laminin & Basement membrane & $100 \%$ & $100 \%$ & - & - & NS & 30 \\
\hline Fibronectin & Extracellular matrix & $100 \%$ & $100 \%$ & - & - & NS & 30 \\
\hline
\end{tabular}

was detected in $80.0 \%$ of non-infected tissue, with similar expression $(72.5 \%)$ in the infected biopsies (NS). Subsequently we assessed the expression of E-cadherin in both tissue groups. The majority of non-infected tissue samples $(83.2 \%)$ showed very strong staining for this molecule, distributed mainly in the basolateral surface of the epithelial cells, while strong staining was only observed in only $25.0 \%$ of the infected samples. Statistical analysis by $\chi^{2}$ test showed that the numbers of infected patients showing reduced expression of E-cadherin were increased $(\mathrm{p}<0.001, \mathrm{n}=30)$ compared with non-infected individuals (fig $1 \mathrm{E}$ and $\mathrm{F}$ ). Semiquantitative data are given in table 1 .

\section{Discussion}

The importance of cellular adhesion in the progression of a malignant neoplastic process has long been accepted. Moreover, the morphogenesis of normal and transformed cells is in part governed by the functional expression of cell adhesion molecules. ${ }^{12}$ In particular, the protein E-cadherin is essential for maintaining cell to cell adhesion, as well as for differentiation, tissue structure, and epithelial polarisation, ${ }^{8}$ and is though to act as an important suppressor of epithelial tumour cell invasiveness and metastasis. ${ }^{9}$

In this study we have shown that epithelial E-cadherin expression is significantly reduced in $H$ pylor $i$ infected gastric tissue. It is accepted that patients infected with $H$ pylori have up to a ninefold greater risk of developing gastric cancer. ${ }^{13}$ In this regard, E-cadherin downregulation could be related to $H$ pylori induced epithelial cell hyperproliferation, ${ }^{5}$ as a decrease in its expression would facilitate epithelial cell proliferation and tumour spread. Downregulation of E-cadherin has also been described in other gastrointestinal diseases, such us Barrett's oesophagus, oesophageal adenocarcinoma, ${ }^{14}$ and in epithelium adjacent to ulcers. ${ }^{15}$ However, E-cadherin has been reported to be strongly and evenly expressed by the epithelium both in normal intestine and in inflammatory conditions. ${ }^{15}$

The expression of laminin, collagen IV, and fibronectin-components of epithelial basement membranes and extracellular matrix, respectively-was not influenced by the presence of $H$ pylori, neither did the infection influence the expression of $\alpha 6$-integrin, an essential protein in epithelial cell binding to the basal lamina. Therefore ulceration of the gastroduodenal mucosa, so strongly associated with $H$ pylori colonisation, ${ }^{1}$ does not seem to correlate with disturbances at the level of the basal lamina or the binding functions mediated by $\alpha 6$-integrin.

In conclusion, we have shown that $H$ pylori infection is significantly associated with downregulation of E-cadherin, an essential protein for the maintenance of solid tissues and for differentiation. However, the infection is not associated with changes in the expression of a6-integrin, collagen IV, laminin, or fibronectin.

We wish to thank Dr Sánchez-Madrid for the kind gift of the anti- $\alpha 6$-integrin antibody. This study was supported in part by grants from the Fumadihe (Spain) and Ministry of Health (Spain, FISS 95/265). DK is a Wellcome senior fellow in clinical science.

1 Cover T, Blaser M. Helicobacter pylori and gastroduodenal disease. Annu Rev Med 1992;43:135-45.

2 Correa P. Helicobacter pylori and gastric carcinogenesis. Am 7 Surg Pathol 1995;19(suppl 1):S37-43.

3 Borén T, Falk P, Roth K, et al. Attachment of Helicobacter pylori to human gastric epithelium mediated by blood group antigens. Science 1993;262:1892-5.

4 Trust T, Doig P, Emody L, et al. High-affinity binding of the basement membrane proteins collagen type IV and laminin to the gastric pathogen Helicobacter pylori. Infect Immun 1991;59:4398-404.

5 Fan XG, Kelleher D, Fan XJ, et al. Helicobacter pylori increases proliferation of gastric epithelial cells. Gut 1996;38:19-22.

6 Adams J, Watt F. Regulation of development and differentiation by the extracellular matrix. Development 1993;117: 1183-98.

7 Simon-Assman P, Kedinger M, Haffen K. Immunocytochemical localization of extracellular-matrix proteins in relation to rat intestinal morphogenesis. Differentiation 1986;32:59-66.

8 Garrod D, Collins J. Intercellular junctions and cell adhesion in epithelial cells. In: Fleming TP, ed. Epithelial organization and development. London: Chapman and Hall, 1992:1-53.

9 Birchmeier W, Beherens J. Cadherin expression in carcinomas: role in the formation of cell junctions and the prevention of invasiveness. Biochim Biophys Acta 1994; 1198:11-26.

10 García-Monzón C, Moreno-Otero R, Pajares JM, et al. Expression of a novel activation antigen on intrahepatic CD8+ T lymphocytes in viral chronic active hepatitis. Gastroenterology 1990;98:1029-35.

11 Sternberger LA. Immunocytochemistry, 2nd ed. New York: John Wiley and Sons, 1979

12 Takeichi M. The cadherins: cell-cell adhesion molecules controlling animal morphogenesis. Development 1988;102: 639-55.

13 Schistosomiasis, liver flukes, and Helicobacter pylori. In: IARC monographs on the evaluation of carcinogenic risks to humans. Lyon, France: IARC, 1994:177-220.

14 Swami S, Kumble S, Triadafilopoulos G. E-cadherin expression in gastroesophageal reflux disease, Barrett's histochemical and immunoblot study. Am $\mathcal{f}$ Gastroenterol histochemical and

15 Dogan A, Wang ZD, Spencer J. E-cadherin expression in intestinal epithelium. f Clin Pathol 1995;48:143-6. 